\title{
Wird die Menschheit kränker oder die Krankheit menschlicher?
}

\author{
Does Humanity Become More III or Illness More Human?
}

Autor

Institut
Thomas Bock

Zentrum für Psychosoziale Medizin, Irre menschlich Hamburg, Universitätsklinikum Hamburg-Eppendorf

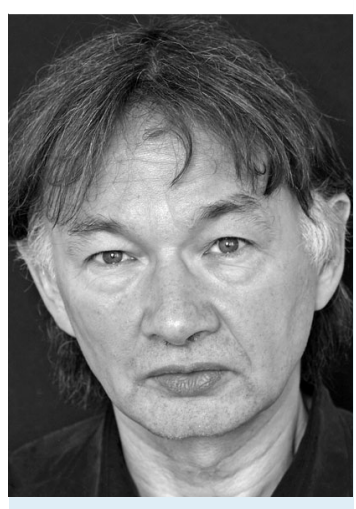

Prof. Dr. Thomas Bock

\section{Bibliografie}

Dol http://dx.doi.org/ 10.1055/s-0034-1369919 Psychiat Prax 2014; 41:

121-123

(c) Georg Thieme Verlag KG

Stuttgart · New York

ISSN 0303-4259

Korrespondenzadresse

Prof. Dr. Thomas Bock

Zentrum für Psychosoziale

Medizin, Irre menschlich

Hamburg, Universitätsklinikum

Hamburg-Eppendorf

Martinistraße 52

20246 Hamburg

bock@uke.de
Liest man die drastischen Zahlen in der Presse über die Zunahme von Krankschreibungen wegen psychischer Erkrankung, kann einem angst und bange werden. Interpretiert man die Zahlen als wachsende Bereitschaft, sich zu einer psychischen Erkrankung zu bekennen, erscheint möglich, dass der Kampf gegen Vorurteile doch langsam Fortschritte macht. In beiden Fällen wird die Struktur der Versorgung der Entwicklung nicht gerecht, verlangen innovative Ansätze mehr Beachtung [1].

\section{Epidemiologische Sicht \\ $\nabla$}

Aktuelle Daten gehen von einer 12-Monats-Prävalenz von 27,7\% aus [2]. Damit sind unterschiedslos alle psychischen Erkrankungen gemeint. Experten erklären diese Zahlen nicht mit einer Zunahme schwerer Störungen, sondern mit einer größeren Bereitschaft von Behandlern und Behandelten, andere oder sich als psychisch erkrankt zu bezeichnen [3]. Sicher scheint, dass Menschen früher erkranken oder früher als krank wahrgenommen werden und dass sie älter werden, also auch länger krank bleiben und häufiger dement werden. Sicher werden mehr körperliche Leiden inzwischen als psychosomatisch diagnostiziert und es gibt auch sonst Verschiebungen zwischen den Diagnosen - aktuell womöglich zwischen Borderline- und Bipolarer Störung. Sicher ist, dass sich die Geschlechter annähern: Mehr Männer werden depressiv, mehr Frauen süchtig - oder eben entsprechend wahrgenommen. Die größere Nachfrage führt angesichts der bekannten strukturellen Mängel [4] zu einer zunehmenden Benachteiligung der Menschen mit ernsten und anhaltenden psychischen Störungen $[1,3]$.

\section{Sozialepidemiologie \\ $\nabla$}

Selbstverständlich haben die Lebensbedingungen eines Menschen Einfluss auf seine seelische Gesundheit und auf seine Ressourcen zur Abwehr von Erkrankung. Nicht nur die berühmten alten, sondern viele neue Studien belegen die Relevanz sozialer Faktoren wie Arbeitslosigkeit oder prekäre Lebensbedingungen für Häufigkeit und Verlauf psychischer Erkrankungen [4, 5] - leider bisher weitgehend ohne Konsequenzen für die Verteilung von Ressourcen oder gar für Prävention. Hamburg gilt als die Stadt mit den meisten Krankschreibungen aus psychischen Gründen. Auch deshalb, weil es mehr Fachleute gibt, die davon profitieren? Zugleich liegt Hamburg an erster Stelle auf dem sogenannten „Glücksatlas“, der mit gemischten Daten Lebenszufriedenheit zu erfassen sucht. Wie passt das zusammen? Wir wissen, dass soziale Ungleichheit ein Indikator für psychische Erkrankung ist [6]. Aber kann es vielleicht auch sein, dass in einem Leben beides Platz hat - Zeiten psychischer Erkrankung und Momente des Glücks? Entscheidend dafür sind kulturelle Akzeptanz und Vorurteilsfreiheit in Psychiatrie und Gesellschaft [7].

\section{Krankheitsverständnis im Interessen- konflikt \\ $\nabla$}

Ob und wie psychische Erkrankungen wahrgenommen werden, ist nicht nur für den Betroffenen, Angehörigen und Behandler relevant, sondern auch für Leistungs- und Kostenträger, Verbände, Organisationen, ganze Wissenschaftsund Industriezweige, Aktionäre und Lobbyisten. Spätestens die Diskussion um DSM-5 hat das unübersehbar gemacht. Führende Wissenschaftler warnen vor der grenzenlosen Expansion der Psychiatrie: Eine Trauerreaktion, die länger als zwei Wochen dauert, ein rebellisches Kind, ein ver- 
gesslicher Erwachsener - die Psychiatrie verliere ihre Verhältnismäßigkeit, vergesse die anthropologischen und kulturellen $\mathrm{Zu}-$ sammenhänge menschlichen Leidens und unterwerfe sich selbst einseitigen Interessen der Pharmaindustrie [8]. Doch diese Debatte zum Verständnis psychischer Erkrankung darf sich nicht auf die Grenzbereiche beschränken. Alle psychischen Störungen haben anthropologische Aspekte. Auch Psychoseerfahrene leiden, wenn ihre besondere Erfahrung auf einen Defekt reduziert wird. Gerade sie brauchen Respekt gegenüber ihrer ganzen Person und umfassende Hilfe bei der Aneignung des Erlebten.

\section{Wer stigmatisiert wen und warum? \\ $\nabla$}

Wer Stigmatisierung soziologisch nüchtern als „Zuordnung negativ bewerteter Merkmale“ begreift, erkennt den Ort des Geschehens: Wir Psychiatrie-Profis stigmatisieren, ob wir wollen oder nicht. Also tragen wir auch Verantwortung für die Wirkung unserer Worte. Entsprechend wichtig ist unsere Fähigkeit zu übersetzen und für Akzeptanz zu sorgen [9, 10]. Die Vorurteile von heute sind die Fehler der Psychiatrie von gestern. Die Annahmen von Unheilbarkeit, von Gefährlichkeit, von der Schuld der Mütter verweisen auf entsprechende inzwischen überholte Konzepte. Insofern müssen wir dem möglichen Vorurteil von morgen, psychische Störungen seien ausschließlich Fehlleistungen des Gehirns, schon heute entgegenwirken.

\section{Wie gesund ist krank?}

Pathologie betont den Unterschied, die Besonderheit, die statistische Normabweichung. Die anthropologische Sicht sucht auch im Besonderen das Allgemeine, auch im Kranken das Menschliche, auch im Störenden die subjektive Bedeutung. Niemand ist nur krank, niemand völlig gesund. Beide Sichtweisen ergänzen sich und schützen einander vor Überzeichnung. Die folgende Übersicht kann nur eine Ahnung geben [1, 9]:

- Angst ist zunächst einmal keine Erkrankung, sondern eine überlebenswichtige Fähigkeit des Menschen (des Kindes, der Menschheit), um sich vor Gefahr zu schützen. Erst die Generalisierung und absolute Zuspitzung markiert den Übergang zur Erkrankung.

- Zwangshandlungen im Sinne von Ritualen können, wenn sie kulturell eingebunden sind, Angst beherrschen helfen. Alle Religionen kennen diesen Zusammenhang. Erst wenn diese Einbindung fehlt und eine oft beträchtliche Eigendynamik einsetzt, beginnt der Übergang zur Erkrankung.

- Auch Depressionen sind zunächst ein Schutzmechanismus der Seele, eine Art Totstellreflex in Momenten der Überforderung durch extreme Erfahrungen oder widersprüchliche Gefühle. Die anschließend psychische, soziale und somatische Eigendynamik birgt hohe Risiken; doch die Funktionalität ganz zu leugnen, macht therapeutisch wenig Sinn. Das gilt auch für die mögliche „Flucht nach vorne“ heraus aus der Überanpassung in Richtung Manie. Erst wenn das Zeitgefühl verlorengeht und Depression und Manie als ewig erscheinen, steigen Verzweiflung bzw. Leichtsinn ins Unendliche.

- Borderline-Patienten erscheinen anstrengend, gewissermaßen als Beziehungsextremisten, haben aber zugleich auch extrem feine Antennen für andere. Die Spannungsfelder zwischen Nähe und Distanz, Autonomie und Bindung, Anpassung und Widerstand, in denen sie sich zu zerreißen drohen, sind eine Herausforderung für jeden Menschen. Nur manchen gelingt die Balance leichter oder sie können sie auf die Pubertät begrenzen.

- Psychotisch werden kann jeder, durch Überreizung oder Isolation; nur die Schwelle ist verschieden. Wer psychotisch wird, steigt aus der als gemeinsam angenommenen Realität aus. Zugleich greift er auf frühe Wahrnehmungswelten zurück: Wenn Kinder alles auf sich beziehen, dann weil sie noch nicht von sich abstrahieren und die Welt nicht anders ordnen können. Auch später geschieht das nicht grundlos; die Zusammenhänge zu verstehen, kann helfen einen Ausweg zu finden. Auch die subjektiven und physiologischen Parallelen zwischen Psychose und Traum sind praxisrelevant: So wie es Alb- und Wunschträume gibt, hat auch die Psychose Angst- und Wunschanteile: Wer z. B. von drei Geheimdiensten verfolgt wird, hat nicht nur Angst, sondern auch Bedeutung [10].

\section{Hunger nach Bedeutung}

$\nabla$

Gerade Menschen mit psychischen Störungen haben einen Hunger nach Bedeutung. Es reicht nicht, sie zu braven Hilfeempfänger zu machen - eine Herausforderung für Sozialpsychiatrie und eine Begründung für Inklusion. Die anthropologische Sichtweise will nicht verharmlosen, schon gar nicht Erkrankung leugnen; sie will den Blick für Ressourcen öffnen und Angst reduzieren - bei allen Beteiligten. Diese Sichtweise hat in Deutschland eine längere Geschichte, hat Wurzeln in der Phänomenologie Karl Jaspers [11], der Daseinsanalyse Binswangers [12] und der viel zu schnell vergessenen Philosophie Kiskers [13]. Sie gewinnt Kraft durch den Trialog [14], das Erstarken der Erfahrenen und Angehörigen sowie die UN-Konvention, perspektivisch auch durch die Neuorientierung der Psychotherapie - z.B. schulenübergreifend im Dachverband Psychosenpsychotherapie [15].

\section{Sinn-Bedürfnis \\ $\nabla$}

Reduktionismus lohnt nicht: Folgt man aktuellen Metaanalysen [16] nahmen Vorurteile der Deutschen Bevölkerung gegenüber Menschen mit der Diagnose Schizophrenie über die letzten Jahre nicht ab, sondern korrespondierend mit biologischen Krankheitskonzepten sogar zu. Doch nicht nur die soziale Distanz Dritter wird durch enge Krankheitskonzepte verstärkt, sondern auch die innere Distanz der Betroffenen. Die Annahme eines fließenden Übergangs zwischen gesund und krank hilft gegen Selbstund Fremdstigmatisierung [16]. Auf beiden Ebenen müssen wir auf Reduktionismen verzichten: Wenn wir dem Sinnbedürfnis der Erfahrenen entsprechen und die Aneignung der widersprüchlichen Erfahrung unterstützen, erhöhen wir die Chancen auf Kohärenz und Genesung [17, 18]. Auf der gesellschaftlichen Ebene fördern Begegnungsprojekte von trialogischen Bürgerinitiativen, wie „Irre menschlich Hamburg“ Toleranz und Sensibilität im Umgang mit anderen und mit sich - ein Beispiel für Prävention [19]. 


\section{Gesund mit Krankheit leben - Mehrwert der Peerberatung \\ $\nabla$}

Psychiatrie darf sich nicht auf Symptombekämpfung reduzieren. Sie muss Genesung begleiten - mit strukturübergreifender Kontinuität. Die Idee einer Orientierung auf Gesundheit/Recovery [7] kann viele Reformer vereinen. Sie bekommt Unterstützung zur rechten Zeit: In Gestalt der Experienced-Involvement-Fortbildung und der anschließenden Peerberatung kehrt der Trialog in die Psychiatrie zurück. Beispielhaft im Hamburger Psychenetprojekt erhalten Betroffene und Angehörige eigenständige Peerberatung in allen Kliniken [20]. Ein innovatives Angebot, das mehrfach Wirkung zeigt: Die Berater wachsen an ihren Aufgaben; die Psychiatrie lernt umzudenken, und die Patienten erhalten eine Hilfe auf Augenhöhe, erleben Ermutigung und entwickeln Selbstvertrauen [21]. Skeptiker der Peerarbeit seien daran erinnert, dass mehrere Wissenschaftler, die hochaktuelle therapeutische Konzepte entwickelten, dies auch auf der Basis eigener Krankheitserfahrung taten - z.B. die Borderline-Forscherin Marsha Linehan und die selbst bipolare Professorin Kay R. Jamison $[22,23]$.

\section{Ausblick}

Eine Psychiatrie, die immer mehr Störungsbereiche für sich reklamiert, um diese mit immer engeren Verstehensmodellen und Konzepten zu beantworten, muss in einer Legitimationskrise landen [6]. Die heutigen Lebensbedingungen bergen besondere Risiken; doch die sind nicht mit einer Ausweitung der Psychiatrie zu lösen, sondern erinnern an unsere politische Verantwortung. Die aufsuchenden Konzepte der Integrierten Versorgung erfordern ein offenes Verständnis seelischer Not und eine Dialogfähigkeit der Therapeuten [1]. Prävention erfordert eine politische Besinnung auf die Folgen von Gewalt, Arbeitslosigkeit und sozialer Ungleichheit. [6]. Die Lebenssituation längerfristig psychisch beeinträchtigter Menschen lässt sich nur im Kontext von Sozialraum und Kommunalpolitik verbessern. Psychische Erkrankung hat neben der psychischen, somatischen und sozialen auch eine gesellschaftliche Dimension. Im gesellschaftlichen Kontext spiegelt der Schuldwahn der einen die Verantwortungslosigkeit der anderen. Und manche Paranoia wird von der NSA-Wirklichkeit eingeholt. Die von Allan Francis [8] angestoßene Debatte um das Ausufern der Psychiatrie bedarf einer Ergänzung: Auch im Kernbereich der Psychiatrie braucht die Pathologie ein anthropologisches Gegengewicht. Die Grenzen zwischen Normalität und Krankheit sind für alle Diagnosen fließend - und das ist gut so.

\section{Literatur}

1 Bock T. Psychiatrie im Widerstreit der Interessen - Risiken und Chancen für die Zukunft. Forum für Gemeindepsychologie 2013; 18: 3

2 Jacobi F, Höfler M, Strehle J et al. Psychische Störungen in der Allgemeinbevölkerung. Nervenarzt 2014; 85: 77-87

3 Wittchen HU. Werden wir alle verrückt? Zur Entwicklung und Wahrnehmung psychischer Erkrankungen. Vortrag vom 9.1.2014 im Dresdner Hygiene-Museum zum Thema Innensichten psychischer Erkrankungen

4 Becker T. Impulsvortrag: Soziale Ungleichheit und seelische Gesundheit, Subjektive Seite der Schizophrenie. Tagung Stralsund 2012

5 O'Campo P, Dunn JR, eds. Rethinking Social Epidemiology - Towards a Science of Change. Dordrecht: Springer; 2012

6 Priebe S, Burns T, Craig TK. The future of academic psychiatriy may be social. Br J Psychiatry 2013; 202: 319-320

7 Amering M, Schmolke M. Recovery. Das Ende der Unheilbarkeit. Bonn: Psychiatrie-Verlag; 2007

8 Francis A. Normal - gegen die Inflation psychiatrischer Diagnosen. Köln: Dumont-Verlag; 2013

9 Bock T. Krankheitsverständnis - zwischen Stigmatisierung und Empowerment. Schweizer Archiv für Neurologie und Psychiatrie 2012; 163 : $138-144$

10 AG Psychoseseminare, Hrsg. „Es ist normal, verschieden zu sein!“ 2007: Im Internet: http://www.lpen-online.de/pdf/es_ist_normal_ver schieden_zu_sein.pdf (Stand: 28.02.2014)

11 Jaspers K. Allgemeine Psychopathologie. Ein Leitfaden für Studierende, Ärzte und Psychologen. Berlin: Julius Springer; 1913

12 Binswanger L. Ausgewählte Vorträge und Aufsätze. Band I. Bern: Fracke; 1947: 217

13 Kisker KP. Der Erlebniswandel des Schizophrenen. Ein psychopathologischer Beitrag zur Psychonomie schizophrener Grundsituationen. Berlin: Springer Verlag; 1960

14 Bock T, Buck D, Meyer H-J. Entwicklungslinien des Trialogs. Sozialpsychiatrischen Informationen 2009; 39: 4-6

15 Dachverband Psychosenpsychotherapie. Im Internet: www.ddpp.eu (Stand: 28.02.2014)

16 Schomerus G, Matschinger H, Angermeyer MC. Causal beliefs of the public and social acceptance of persons with mental illness: a comparative analysis of schizophrenia, depression and alcohol dependence. Psychol Med 2014; 44: $303-314$

17 Bock T, Brysinski T, Klapheck K et al. Zum Subjektiven Sinn von Psychosen - das Hamburger SuSi-Projekt. Psychiat Prax 2010; 37: 285-291

18 Bock T, Klapheck K, Ruppelt F. Sinnsuche und Genesung - Erfahrungen und Forschungen zum subjektiven Sinn von Psychosen (das Hamburger SuSi-Projekt). Köln: Psychiatrie Verlag; 2014

19 Irre menschlich Hamburg e.V. Im Internet: http://www.inno-serv.eu/ content/changing-perceptions-people-mental-illnesses-irre-mensch lich (Stand: 28.02.2014)

20 Bock T, Mahlke C, Schulz G et al. Eigensinn und Psychose, Peerberatung und Psychotherapie. Psychotherapeut 2013; 58: 364-370

21 Daniels AS, Bergeson S, Fricks $L$ et al. Pillars of peer support: advancing the role of peer support specialists in promoting recovery. J Ment Health Train Education Practice 2012; 7: 60-69

22 Carey B. Expert on mental illness reveals her own fight. New York Times 23.06. 2011: A1

23 Jamison KR. An unquiet mind. New York: Vintage; 1996 